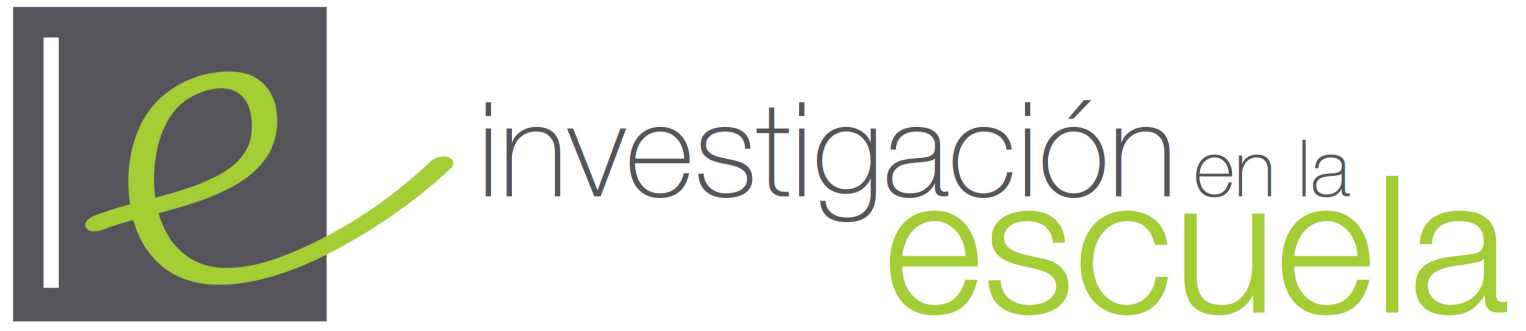

Revista internacional de investigación e innovación educativa

\title{
Comunicación, entendimiento y aprendizaje en grupos interactivos
}

\author{
Carmen Álvarez-Álvarez. \\ Universidad de Cantabria \\ España
}

Citación: Álvarez-Álvarez, C. (2017). Comunicación, entendimiento y aprendizaje en grupos interactivos. Investigación en la Escuela, 91, 90-105. Recuperado de

http:/ /www.investigacionenlaescuela.es/articulos/R91/R91-6

Resumen: Cada vez más escuelas de todo el mundo se interesan por el desarrollo de grupos interactivos, una práctica basada en las teorías y los principios teóricos del aprendizaje dialógico. Presentamos los resultados de una investigación etnográfica basada en la observación participante en tres contextos donde se desarrolla esta práctica innovadora, dos en España y uno en Brasil. Estos resultados muestran que, ante la tarea de resolver un ejercicio en grupos interactivos, el alumnado comunica sus experiencias de aprendizaje previas, negocia posibles vías de solución buscando el acuerdo y aprenden con profundidad. Las conclusiones enfatizan que esta práctica logra favorecer habilidades sociales como el diálogo y el acuerdo, aprendizajes duraderos y valores, dado su desarrollo en un contexto de diversidad y solidaridad.

Palabras clave: "Aprendizaje"; "interacción verbal"; "lenguaje”; "aprendizaje en grupo".

\section{Communication, understanding and learning in interactive groups}

Abstract: More and more schools around the world are interested in developing interactive groups, a practice based on the theories and theoretical principles of dialogic learning. We present the results of an ethnographic research based on participant observation in three contexts where this innovative 
practice, two in Spain and one in Brazil, develops. The results show that, given the task of solving an exercise in interactive groups, students communicate their previous learning experiences, negotiate possible solutions seeking the agreement and learn in depth. The findings emphasize that this practice succeeds valuable social skills such as dialogue and agreement, lasting learning and values, since its development in a context of diversity and solidarity.

Key words: "Learning"; "verbal interaction”; “communication”; "group learning".

\section{Communication, compréhension et apprentissage en groupes interactifs}

Resumè: De plus en plus des écoles de tout le monde sont intéressées par le développement de groupes interactifs, une pratique fondée sur les théories et les principes théoriques de l'apprentissage dialogique. Nous présentons les résultats d'une recherche ethnographique basée sur l'observation participante dans trois contextes où se développe cette pratique innovante, deux en Espagne et un au Brésil. Les résultats montrent que, face à la tâche de résoudre un exercice en groupes interactifs, les étudiants communiquent leurs expériences antérieures de l'apprentissage, des manières possibles de solution qui cherchent l'accord de négociation et apprennent en profondeur. Les conclusions soulignent que cette pratique peut favoriser des compétences sociales telles que le dialogue et l'accord, l'apprentissage durable et les valeurs, compte tenu de son développement dans un contexte de diversité et de solidarité.

Mostsclé: "Acquisition de connaissances"; "verbal interaction"; "communication"; "pédagogie de groupe".

\section{Introducción}

La innovación escolar es el proceso de cambio institucional planificado de un centro educativo para perseguir la mejora (Paredes y de la Herrán, 2009; Lukacs y Galluzzo, 2014). Una práctica que está despertando mucho interés actualmente son los grupos interactivos ya que genera mejoras en, al menos, los siguientes cinco ámbitos: los resultados de aprendizaje, la convivencia, la inclusión, la motivación por el conocimiento y, finalmente, la participación de la comunidad educativa (Valls y Kyriakides, 2013; Flecha y Soler, 2013; Álvarez y Puigdellívol, 2014). En la actualidad los grupos interactivos se desarrollan en más de trescientos centros en España, Brasil, México, Colombia, Chile, Uruguay y Perú y han pasado a ser considerados una actuación educativa de éxito (Ministerio de Educación, 2011), surgida en el proyecto de Comunidades de Aprendizaje (Elboj, Puigdellívol, Soler y Valls, 2006) y que se desarrolla en muchos centros educativos comprometidos con la mejora del aprendizaje a través de la participación de la comunidad (Mello, 2009; Flecha, 2009; Díez, Gatt y Racionero, 2011; Gatt, Ojala y Soler, 2011).

Dicha práctica consiste en organizar el alumnado en pequeños grupos heterogéneos (sexo, cultura, edad, nivel de conocimientos, habilidades) para realizar actividades de aprendizaje con la ayuda de una persona voluntaria, que apoya y estimula el trabajo de cada pequeño grupo (Elboj y Niemelä, 2010). Por lo general, en cada aula se forman cuatro grupos y se asigna un voluntario o voluntaria a cada uno, que atiende una actividad planificada previamente diseñada por el maestro/atutor/a, de una duración de entre quince y veinte minutos. Los grupos van rotando por los distintos voluntarios y voluntarias de modo que al final de la clase han podido llevar a cabo las cuatro actividades programadas de repaso en un área relevante (Vieira y Puigdellívol, 2013). Puede ser voluntaria cualquier persona que lo desee, incluyendo los propios familiares, siempre y cuando sigan unos principios básicos dirigidos a favorecer el diálogo igualitario entre el alumnado y a estimular relaciones de ayuda entre los componentes del grupo: diálogo igualitario, inteligencia cultural, transformación, dimensión instrumental, creación de sentido, solidaridad e igualdad de diferencias (Ferrada y Flecha, 2008). El objetivo es que todos y todas realicen la tarea y la entiendan (Racionero, 
2010). Las personas voluntarias no tienen la misión de enseñar, ya que el alumnado está familiarizado con la mayoría de las tareas que se llevan a cabo, sino de apoyar al grupo estimulando la participación de todos y todas, propiciando la ayuda entre iguales y resolviendo los pequeños problemas que puedan surgir con la actividad.

Los grupos interactivos se fundamentan en las perspectivas socio-cultural y dialógica del aprendizaje (Álvarez y Puigdellívol, 2014). La primera nos muestra que el aprendizaje se da en situaciones de interacción en las que primero aparece en el plano inter-individual para, posteriormente, interiorizarse como una adquisición en el plano intra-individual: es entonces cuando el/la estudiante ha alcanzado las competencias necesarias para resolver sin ayuda los problemas planteados por el aprendizaje que se ha adquirido (Vigotsky, 1979). Desde esta perspectiva, por ejemplo, se asevera que el niño aprende a sumar porque ha visto sumar a otros: sólo así llega a interiorizarlo, después de una interacción y una socialización sobre el contenido a aprender. De ahí la importancia de que los grupos sean heterogéneos y de la participación de personas adultas en estas actividades de aprendizaje. El voluntariado suele estar formado por familiares, estudiantes, exalumnado y otros miembros de la comunidad. Muchas de estas personas carecen de un nivel de estudios elevado, pero todas enriquecen las interacciones que se dan dentro del aula: madres, abuelos, otros maestros, miembros de asociaciones del barrio, etc. (Vieira y Puigdellívol, 2013). Además, la presencia del voluntariado incorpora a la escuela el capital cultural de la comunidad a través de otros saberes relevantes y valiosos para la escuela: tradiciones del lugar, valores, ejemplos próximos al contexto cotidiano de los niños, etc. (González y Moll, 2002). El compromiso del voluntariado genera sinergias que favorecen el acercamiento entre la escuela y su entorno, hecho particularmente destacable cuando en dicho entorno predominan las familias "no académicas" y, más aún, cuando se trata de grupos socialmente vulnerables (Díez, Gatt y Racionero, 2011). Muchas investigaciones han constatado la influencia de la implicación de las familias y la comunidad en el éxito académico del alumnado y en su estímulo para el aprendizaje (Brooks 2009; Kyle 2012; BenetConroy 2013; Durand y Pérez 2013; García y Ríos, 2014).

Pero lo que más diferencia a los grupos interactivos de otras prácticas docentes es su fundamentación en la concepción dialógica del aprendizaje (Flecha, 1997; Aubert, Flecha, García, Flecha y Racionero, 2008) que considera el diálogo, la argumentación y la diversificación de las interacciones como el marco idóneo para estimular el aprendizaje de todos y todas (Mercer y Howe, 2012). Son las interacciones comunicativas las que producen el aprendizaje de las personas, de modo que en las aulas es necesario estimular al máximo las mismas entre los niños para potenciar su desarrollo. Aunque desde perspectivas limitadamente técnicas a veces se olvide, todas las personas, con independencia de su nivel académico, pueden hacer aportaciones relevantes a los grupos interactivos y a las demás formas de participación en la escuela, pues todos y todas disponemos de lenguaje, de capacidad de argumentación (Habermas 1987) y de un patrimonio cultural que ofrecer. El hecho de que la comunidad se vea reflejada en el aula, con sus diferentes formas de pensamiento y argumentación, e incluso con sus diferentes registros lingüísticos, contribuye a dicho enriquecimiento y mejora la comunicación, la comprensión y el sentido de pertinencia de todos los miembros del grupo. Para ello es necesario no perder de vista que la finalidad compartida, la principal prioridad de la escuela, es el aprendizaje y el acceso del alumnado, sin excepciones, a los conocimientos y competencias que les permitan alcanzar el éxito académico y el crecimiento personal y como grupo.

Hay un amplio corpus de investigaciones que avala la relevancia de optar por metodologías dialógicas que den la palabra a los sujetos en formación, superando concepciones pedagógicas "bancarias" (Freire, 2003). Sin embargo, los docentes tienden a reproducir las prácticas bancarias o meramente transmisivas por la falta de referencias de prácticas dialógicas (Bosacki, Rose-Krasnor y Coplan, 2014) y por el miedo a que puedan darse situaciones conflictivas (Bickmore y Parker (2014). 
La investigación ha demostrado que cuando hay interacción dialógica entre los participantes, el aprendizaje es más eficaz (Wells y Arauz, 2006); se desarrollan habilidades para pensar, cuestionar y construir conjuntamente conocimientos (Teo, 2013); para hablar y escuchar (Remedios, Clarke y Hawthorne, 2012); para aprender a esquematizar y argumentar (Reznitskaya, Kuo, Clark, Miller, Jadallah, Anderson y Nguyen-Jahiel, 2009); y para razonar críticamente e interiorizar el debate (Gillies, 2014). Además, la interacción dialógica también promueve mejores climas de aula y el desarrollo de comportamientos colaborativos y valores solidarios (Christie, Tolmie, Thurston, Howe y Topping, 2009; Remedios, Clarke y Hawthorne, 2012).

En lo que respecta a la investigación específica sobre grupos interactivos, en los últimos años han comenzado a realizarse estudios al respecto. Chocarro y Sáenz (2016) han hecho grupos de discusión con profesorado, voluntariado y alumnado para valorar la implementación de grupos interactivos durante un curso y han concluido que esta forma de trabajo produce: una mayor implicación del alumnado en su aprendizaje, un creciente compromiso con la tarea motivado por la dinámica de la misma, aprendizaje con los otros a través de las interacciones y mejoras convivenciales.

Gómez, Aguilera y Prados (2016) han analizado 23 informes de estudiantes universitarios que han colaborado con centros educativos sevillanos que implementan grupos interactivos para conocer su valoración de esta propuesta, saber cómo se han sentido y para qué les ha servido esta experiencia, los aspectos positivos y negativos a destacar, etc. y han verificado que la experiencia es muy positiva: les ayuda a superar prejuicios y creer que el cambio social es posible, adquirir experiencia profesional y laboral, superar estereotipos, cambiar percepciones sobre el trabajo escolar (colaboración, participación, mejora del rendimiento, etc.).

Muntaner, Pinya y De la Iglesia (2015) han realizado un estudio de caso único en un centro educativo analizando los resultados de la implementación de grupos interactivos desde la perspectiva de la inclusión durante la programación de actividades, su implementación y el cierre y evaluación, señalando numerosas necesidades de mejora para desarrollar al máximo el potencial de esta forma de enseñanza-aprendizaje: mejorar la planificación de las actividades, formar a familias y voluntariado, tener claros los objetivos, aumentar la participación del personal no docente, que las consideraciones que hagan los adultos sean estimulantes, etc.

Lavín (2014) ha analizado el desarrollo de habilidades cognitivas en la asignatura de Ciencias Naturales en estudiantes con alta vulnerabilidad socio-económica en Chile, a través del proyecto denominado "Enlazando mundos". Los resultados muestran que el alumnado de octavo curso básico logran desarrollar las siguientes habilidades cognitivas: adquisición de conocimientos (identificar, recordar), comprender (explicar, prever, predecir, describir), aplicar (poner en práctica conceptos científicos, aplicar y resolver), analizar (analizar, organizar, deducir), sintetizar (exponer, discutir, planificar) y evaluar (evaluar, defender).

Pese a que contamos con algunos estudios recientes en España y Chile sobre grupos interactivos, carecemos de investigaciones con una perspectiva etnográfica, conociendo desde dentro cómo interactúan los niños y niñas que trabajan en grupos interactivos en diferentes contextos. En este artículo analizaremos cómo llegan a acuerdos y cómo aprenden en su proceso de resolución de actividades. Con ello pretendemos constatar si realmente se están dando relaciones libres de dominio, si las decisiones se toman por consenso, en base a argumentos, y si se logra acelerar/optimizar el aprendizaje. 


\section{Método}

En este artículo presentamos los resultados de una investigación etnográfica en la que se analizan los modos de resolver actividades académicas en aulas que desarrollan grupos interactivos en España y Brasil, con niños y niñas en todos los niveles de la etapa de Educación Primaria.

\section{Objetivo}

El objetivo de nuestra investigación es conocer las interacciones comunicativas que se producen entre niños y niñas y personas adultas trabajando en el aula en grupos interactivos. Pretendemos explorar cómo interactúan para aprender en ese proceso de resolución de actividades. Con esta finalidad nos hemos introducido como etnógrafos en el trabajo que desarrollan diferentes grupos interactivos en diferentes contextos y con alumnado de diferentes edades, tomando nota de todas las interacciones que se producen.

\section{Método empleado}

El método de investigación empleado es el estudio de casos (Stake, 2005) y el modelo es la etnografía (Hammersley y Atkinson, 2007). El objetivo último de los estudios de casos es conocer unidades relevantes de análisis para indagar en profundidad a nivel "micro" una realidad, en nuestro caso educativa, y así ofrecer ideas que permitan entenderla, contribuyendo a su mejora. En concreto, al recabar datos en tres contextos, es posible hablar de un estudio multi-caso. La etnografía considera que para conocer bien una realidad es preciso vivirla como lo hace un miembro de la propia comunidad.

\section{Casos}

Hemos participado en condición de observadores en diferentes grupos interactivos en tres centros educativos. Fueron elegidos por un criterio de proximidad. Los denominaremos así:

Centro 1: Escuela en España no transformada en Comunidad de Aprendizaje. Inició su andadura en grupos interactivos en el curso 2010 en dos aulas y actualmente se desarrollan los grupos interactivos en ocho. Se trata de un centro educativo urbano situado en una ciudad dormitorio y de pescadores del norte de España. Está situado en el casco histórico, donde viven en estos momentos la mayor parte de las familias inmigrantes de la ciudad, que envían a sus hijos a esta escuela. Hemos asistido a aulas de todos los niveles educativos. En grupos interactivos se trabajan las materias de lengua y matemáticas. En momentos puntuales han trabajado las materias de ciencias naturales e inglés.

Centro 2: Escuela en Brasil transformada en Comunidad de Aprendizaje en 2005. Fue visitada en el curso 2011-2012. Se trata de un centro educativo urbano situado en una ciudad del interior del Estado de São Paulo. El barrio es uno de los más pobres de la ciudad: exclusión social, familias en paro y sin medios viviendo en chabolas sin luz ni agua, en algunos casos envueltas en actividades ilícitas. Hemos asistido a aulas de dos niveles educativos porque así lo estimó adecuado el centro. En grupos interactivos se trabaja la materia de lengua.

Centro 3: Escuela en España no transformada en Comunidad de Aprendizaje. Inició su andadura en grupos interactivos a finales del curso 2013-2014 en todas sus aulas de Primaria (un total de 8 , es un centro de una línea con dos grupos en dos cursos). Se trata de un centro educativo urbano situado en una capital de provincia del norte de España. Está situado en un barrio de clase obrera y recibe un alto porcentaje de alumnado excluido socialmente: inmigración, necesidades 
educativas especiales y etnia gitana. Hemos asistido a aulas de todos los niveles educativos. En grupos interactivos se trabajan las materias de lengua y matemáticas.

Los tres casos son bastante afines: el contexto de partida es desfavorecido en los centros españoles y muy desfavorecido en el caso brasileño. Sin embargo, y pese a esto, los centros están concienciados en relación con el desarrollo de grupos interactivos en las aulas y con la importancia de la mejora del aprendizaje de los niños, los docentes y los equipos directivos han recibido formación, y cuentan con numerosos voluntarios que acuden al centro semanalmente a colaborar: abuelos, madres, maestros del centro, maestros jubilados, estudiantes universitarios, profesionales del barrio, etc. (Fernández, 2015). Esto genera un estímulo para todos en condiciones tan adversas.

\section{Procedimiento}

Para recoger los datos que configuran este estudio se han visitado los tres centros mencionados a lo largo de los pasados cursos y se han registrado 156 momentos de interacción. En la siguiente tabla pueden observarse todas las visitas realizadas y el número de interacciones recogidas en cada visita.

El observador participaba como observador en el grupo, evitando interaccionar con los niños y el voluntario, tomando notas de las interacciones que se producían para lograr el aprendizaje. El propósito era recoger evidencias de interacción en las que se verificasen situaciones de aprendizaje para explorar cómo interactúan niños y adultos, cómo llegan a acuerdos y cómo aprenden en ese proceso de resolución de actividades.

Para registrar la información recogida en el proceso de visita a los centros hemos categorizado las observaciones en tablas como la siguiente (Tablas 1 y 2).

Tabla 1

$V$ isitas realizadas a los centros e interacciones recogidas

\begin{tabular}{l|l|l|l}
\hline \multicolumn{1}{c|}{ Centros } & \multicolumn{1}{|c|}{ Visitas efectuadas } & Visitas realizadas & Interacciones recogidas \\
\hline \multirow{4}{*}{ Centro 1 } & Curso 2010-2011 & 4 & 14 \\
\cline { 2 - 4 } & Curso 2011-2012 & 3 & 26 \\
\cline { 2 - 4 } & Curso 2012-2013 & 2 & 18 \\
\cline { 2 - 4 } & Curso 2013-2014 & 1 & 5 \\
\cline { 2 - 4 } & Curso 2014-2015 & 3 & 12 \\
\hline Centro 2 & Curso 2011-2012 & 5 & 5 \\
\hline \multirow{2}{*}{ Centro 3 } & Curso 2013-2014 & 1 & 33 \\
\cline { 2 - 4 } & Curso 2014-2015 & 3 & 156 \\
\hline Total & $2010-2015$ & 22 & \\
\hline
\end{tabular}


Tabla 2

Rejilla de observación empleada

\begin{tabular}{l|l}
\hline Código de la interacción & Compuesto por fecha y un número identificativo. \\
\hline Contexto de la interacción & $\begin{array}{l}\text { Curso, perfil de la persona voluntaria y breve descripción } \\
\text { de la tarea. }\end{array}$ \\
\hline Descripción de la interacción & $\begin{array}{l}\text { Con la mayor literalidad se trata de dar cuenta de las } \\
\text { interacciones producidas en el grupo en un momento de } \\
\text { resolución del ejercicio. Para transcribir se emplean las } \\
\text { claves: A1, A2, A3... para las intervenciones del alumnado } \\
\text { y V para las de la persona voluntaria. }\end{array}$ \\
\hline $\begin{array}{l}\text { Relación con los principios } \\
\text { del aprendizaje dialógico }\end{array}$ & $\begin{array}{l}\text { Hemos relacionado estas interacciones transcritas con } \\
\text { principios concretos del aprendizaje dialógico que se } \\
\text { ponen claramente en juego. }\end{array}$ \\
\hline Interpretación personal & $\begin{array}{l}\text { El observador/a muestra su propia visión sobre ese } \\
\text { momento de trabajo concreto. }\end{array}$ \\
\hline
\end{tabular}

A lo largo de estos cinco años, hemos realizado 22 visitas a estos centros para ver cómo trabajaban y aprendían los niños y recabar información para realizar este estudio y hemos obtenido 156 interacciones comunicativas en grupos interactivos, es decir, 156 situaciones diferentes de intercambio oral resolviendo tareas escolares que han sido transcritas y analizadas.

\section{Análisis de datos}

Mientras se producía la recogida de esta información, procedimos a organizar todos los datos en las tablas. Tras esto, procedimos a analizar los datos, resultando tres categorías fundamentales comunes (comunicación, entendimiento y aprendizaje). Seguimos pautas propias del análisis de contenido ya que todos son de tipo cualitativo, dada la necesidad de conocer las interacciones producidas entre los y las participantes en grupos interactivos. En el apartado de resultados se ofrece algún fragmento de trascripción para ilustrar la información recabada.

\section{Resultados}

Los resultados muestran que los niños y niñas que trabajan en grupos interactivos están permanentemente sometidos a procesos de comunicación, entendimiento y aprendizaje. Para dar cuenta de los resultados hallados a estos tres niveles se aportan en cada uno de ellos algunos episodios breves de interacción recogidos que ayudan a ilustrarlos (hemos seleccionado tres de lengua y tres de matemáticas).

\section{Comunicación}

Entendemos por comunicación el proceso de interacción que provoca que los niños y niñas sepan hablar y escuchar en el seno del trabajo de grupo. Veamos el siguiente ejemplo (del Centro 1): 
Tienen una ficha de matemáticas que está pensada para repasar la tabla del 7 que se han estado estudiando en clase. Tienen telarañas y un número de arañas en cada tela para trabajar el cálculo. Cada uno mira la suya y empiezan a hablar.

A1. Esta ficha parece difícil. Yo no la entiendo.

V. Ricardo, explícaselo a Lucas.

A2. Esto tiene que ver con la tabla del 7 que estudiamos. En cada tela hay siete arañas y depende las telas que hay multiplicamos el 7 por uno o por dos o por tres. No es difícil. Mira la primera fila. Hay tres telas y cada tela tiene siete arañas.

A1. ¿ 7 por 3 ?

A2. Sí, ¿cuántas son?

A1. Tengo que calcularlo.

V. Vamos a ir haciéndolos todos juntos. ¿Lo entendiste, Lucas?

A1. Sí, pero no me acuerdo de la tabla del 7. No entendía las telarañas.

V. Vamos a hacer las primeras juntos, entre todos y luego si salen bien seguís vosotros solos. Continúan hablando y resolviendo la ficha en colaboración.

En grupos interactivos, al priorizar el trabajo oral sobre el escrito u otras formas de comunicación, siempre se produce la comunicación el diálogo entre los niños y niñas del grupo y la persona voluntaria. El diálogo permite la comunicación, lo que se puede ver en al menos cinco situaciones del fragmento anterior. Estas cinco situaciones también han aparecido en otras transcripciones de interacciones:

a. Se da una interacción dialógica dirigida a la resolución de la actividad a lo largo de todo el proceso de resolución del ejercicio, desde el principio hasta el final. Se prioriza el intercambio verbal y la exploración conjunta de la actividad.

b. En grupos interactivos se permite la libre expresión. En este ejemplo, hay una afirmación así al inicio: "Esta ficha parece difícil. Yo no la entiendo". Está permitido que los niños y niñas se expresen libremente en todo momento con arreglo al tema a tratar y la actividad a resolver.

c. Se van siguiendo turnos de palabra de manera espontánea, a veces pidiéndola explícitamente y en otras no. En otras ocasiones es la persona voluntaria quien estimula a alguien a intervenir: "Ricardo, explícaselo a Lucas". La persona voluntaria ha detectado que un niño puede ayudar al otro y se lo pide explícitamente mientras el grupo atiende a cómo debe ser resuelto el ejercicio. La ausencia de turnos de palabra para hablar en ocasiones genera dificultades en la comunicación.

d. Poco a poco se va dando una autorregulación y una regulación externa (aspecto que al inicio cuesta un poco). La persona voluntaria y en ocasiones también los compañeros y compañeras ayudan a que cada niño/a se autorregule en el proceso de resolver una actividad. En el ejemplo se ve en la intervención de la persona voluntaria: "Vamos a ir haciéndolos todos juntos". La voluntaria sugiere una forma de trabajo y se cerciora de que el niño con dificultad ha entendido el ejercicio.

e. Algunos niños y niñas asumen un liderazgo momentáneo, como se ve en este caso, cuando Ricardo asume el rol de ayudar a Lucas en la resolución de la actividad. Pero ese liderazgo pueden ejercerlo todos los miembros del grupo a lo largo del desarrollo de los grupos interactivos, puesto que esta forma de trabajo les permite ejercer de enseñantes por momentos con sus propios compañeros y compañeras.

Estas cinco situaciones también han aparecido en las otras interacciones recogidas, como en este ejemplo del Centro 1: 
V. Tenemos una ficha de lectura.

A1. Unos niños van a un bosque.

V. Eso parece.

A1. Es para que sepamos cuidar el bosque y el medio ambiente.

V. Vamos a hacerla.

Leen el texto y luego responden a las preguntas:

V. ¿Por qué no se debe tirar basura al río? Elige la opción correcta.

A2. Las tres son verdaderas. Yo marco las tres.

$\mathrm{V}$. ¿Estáis de acuerdo?

Todos. Sí.

V. Pues marcamos las tres opciones con una crucecita. Fátima, lee la segunda pregunta.

A3. Colorea de rojo la basura del río.

V. Pues con los lápices de colores coloreamos de rojo la basura.

A4. Acabé de colorear. Pasamos a la siguiente.

V. Vale, vamos a hacer la siguiente y luego seguís coloreando las basuras del agua.

A4. ¿Qué se puede hacer y qué no se puede hacer en el bosque?

V. Vamos a hacer una lista a cada lado de la hoja de lo que sí se puede y de lo que no se puede.

Continúan trabajando.

\section{Entendimiento}

Entendemos por entendimiento el proceso de interacción mediante el que los alumnos barajan opciones y toman acuerdos trabajando en grupo. Los grupos interactivos propician que a través de la exploración conjunta los niños y niñas del grupo, con el estímulo de la persona voluntaria, lleguen a acuerdos, logrando un entendimiento basado en la comunicación Con ello se logra un avance ágil y conjunto en la resolución de la tarea. Podríamos hablar de tres características en este proceso de entendimiento:

a. En la comunicación con frecuencia se dan diferentes posibilidades de resolución de un ejercicio y el grupo tiene que sopesar y elegir entre diferentes alternativas a su alcance, como en este ejemplo de interacción en el Centro 3:

El alumnado transcribe el resultado de su ejercicio a la fotocopia y al hacerlo tienen una duda.

A1. Tenemos que escribir este número aquí, pero hay un tres puesto, no sé si tenemos que poner nuestro número debajo o seguido.

V. Aprovechamos ese tres que ya está escrito.

A2. Vale. A borrar y ponerlo bien.

A3. Ah, claro, como en un crucigrama.

A4. Sí.

b. Si es necesario, el grupo argumenta sobre las ventajas e inconvenientes de las diferentes opciones a su disposición y tras escuchar las propuestas se escoge la que consideran mejor. Cuando se dan diferentes alternativas hay miembros del grupo que aprenden a ceder y manifiestan que su propuesta quizás no era la mejor, porque la correcta y rápida resolución de la tarea de grupo es prioritaria sobre la defensa de una postura.

c. Cuando los niños y niñas llevan un tiempo trabajando en grupos interactivos, sorprende comprobar lo rápido que toman acuerdos, algo que en un inicio resulta más costoso, saltándose en 
ocasiones los dos pasos previos señalados como "a" y "b"), ya que rápidamente visualizan las alternativas y tienen claro con cuál quieren quedarse. Además, una vez han adquirido la destreza de trabajar en grupos interactivos, cada vez quieren perder menos el tiempo en sopesar alternativas para priorizar la resolución del ejercicio. Tal es el caso de este ejemplo del Centro 3, donde se percibe que claramente tienen interiorizado que cuando hay que leer un texto lo mejor es que todos y todas tengan la oportunidad de leer un poco.

V. ¿Hay que hacer una ficha?

A1. Sí, hoy toca ésta.

V. ¿Y para qué hacemos esta ficha?

A2. Para aprender. Hay fichas de todo. Hoy es de lengua. También a veces de mate.

V. ¿Hay fichas de todo?

A3. Sí, de todo.

V. Habrá que leerla, ¿no?

A1. Sí.

V. ¿Cómo la leemos?

A2. Nos ponemos cada uno a leerlo y leemos cada uno una vez, como un círculo.

$\mathrm{V}$. ¿Lee un compañero y escuchamos y luego lee otro compañero?

A2. Sí, así, para aprender.

\section{Aprendizaje}

Entendemos por aprendizaje el proceso por el cual los niños y niñas entienden cómo se resuelve un ejercicio, de tal manera que si en el futuro tuvieran que resolver otro de similares características podrían hacerlo autónomamente. Un ejemplo de interacción en grupo en el Centro 3:

Resolviendo un problema de matemáticas:

V: Hay que decidir si más de 20 euros o menos de 20 euros.

A1: Si quiere comprar los patines que cuestan 119 euros y ella tiene 100 es menos de 20 euros.

V: Tú parece que lo tienes claro, pero tu compañera creo que no tanto. ¿'Tú cómo lo ves? A2: No sé.

V: Apoyadla para que sepa hacerlo. Explícaselo.

A1: Pues que si tiene 100 euros y cuestan 19 euros le faltan menos de 20 euros.

$\mathrm{V}$ : Creo que hay que explicarlo de otra forma.

A1 está muy seguro de que lo está resolviendo bien, empieza a contar con los dedos para demostrárselo a la compañera, pero se confunde y le sale 18. Vuelve contar y salen 19. Luego la compañera cuenta con sus dedos.

A2: Claro, 19. De 100 contamos a 119 y hay 19.

$\mathrm{V}:$ ¿19, qué?

A2: Euros.

$\mathrm{V}:$ ¿Euros, qué?

A2: Necesita 19 euros.

$\mathrm{V}:$ ¿Entonces necesita más o menos de 20 euros?

A2: Menos, claro. Ya.

En grupos interactivos, mediante el trabajo oral permanentemente están hablando sobre asuntos que guardan relación con los temas académicos que están tratando en las clases, logrando 
aprender de un modo efectivo, sin dejar a nadie fuera del grupo. Este proceso de aprendizaje se caracteriza por lo siguiente:

a. Los alumnos y alumnas son verdaderamente los protagonistas de su propio proceso de aprendizaje. Desde que empiezan a trabajar en el grupo saben que la resolución de la actividad depende de su esfuerzo y se sienten comprometidos con la tarea. En el ejemplo anterior se ve que algunos saben afrontarla y otros no, situación que se suele dar con frecuencia, dado el carácter heterogéneo de los grupos.

b. Cada persona toma conciencia de sus propios errores, limitaciones y potencialidades dentro del grupo y desde la autocrítica se genera un fuerte sentimiento y deseo de mejora.

c. Los y las estudiantes comparten el reto de resolver una actividad completa y bien en un tiempo limitado y eso les lleva a concentrarse en la tarea y dar prioridad al aprendizaje.

d. Los ejercicios se resuelven entre todos, con inclusión y solidaridad y sin apartar al alumnado que suele mostrar más dificultades o necesidades educativas especiales. En el ejemplo anterior, la voluntaria los insta a ayudar a la niña del grupo que aún no lo ha entendido: "Apoyadla para que sepa hacerlo. Explícaselo".

e. Pueden explicar y re-explicar la actividad a sus compañeros y compañeras o la persona voluntaria tantas veces como sea necesario, ya que han entendido el procedimiento para hacer el ejercicio al resolverlo ellos por sí mismos. Cuando la niña lo entiende, lo verbaliza con naturalidad: "Menos, claro. Ya". Si en ese momento se le hubiese pedido una explicación del proceso habría podido darla.

Véase otro ejemplo de interacción en grupo en el Centro 2:

Se hace una actividad de lengua que consiste en averiguar cuál es la primera sílaba de algunas palabras. Tienen un montón de pequeños cartones en el centro de la mesa y cada compañero coge uno y le lee la palabra que contiene a un compañero. Van acertando la primera sílaba. De repente, un alumno no sabe la respuesta.

A1. Secreto.

A2. Ce.

A1. Casi. No es correcto. (Miran a la persona voluntaria, buscando la respuesta). V. Ayudaos. Yo creo que lo vais a saber resolver.

A1. Si fuese cecreto, sería ce, pero es secreto. (Enfatiza en la ese al pronunciar. La compañera no percibe la diferencia).

V. ¿Qué pensáis los demás?

A3. Yo creo que es se. Secreto. Se.

A4. Yo lo quiero mirar en un diccionario. ¿Puedo?

Lo comprueban. Continúan con el ejercicio.

\section{Discusión}

Los datos recogidos en tres centros educativos de tres contextos diferentes nos han permitido conocer cómo interactúan, cómo llegan a acuerdos y cómo aprenden los y las estudiantes en el proceso de resolución de actividades en grupos interactivos. En este sentido, los estudio multicasos y la perspectiva etnográfica como metodología de investigación proporcionan una mirada sobre los fenómenos educativos de extraordinaria riqueza, al permitir al investigador o investigadora convivir con los protagonistas de los fenómenos reales que se desean investigar, recogiendo 
información de primera mano, participando como uno más, en igualdad de condiciones (Hammersley y Atkinson, 2007).

Haciendo balance de los resultados, se puede concluir lo siguiente:

a. El trabajo en grupos interactivos, al basarse en el aprendizaje dialógico y poner en juego pautas de trabajo basadas en la solidaridad grupal, el valor de la inteligencia cultural de las personas y el diálogo igualitario y argumentativo, logra favorecer habilidades comunicativas de diálogo, elevando la autoestima de los y as participantes que poco a poco van cogiendo confianza para intervenir en el grupo, mostrar sus ideas y contribuir activamente en la resolución de las tareas propuestas (Reznitskaya, Kuo, Clark, Miller, Jadallah, Anderson y Nguyen-Jahiel, 2009; Remedios, Clarke y Hawthorne, 2012; Flecha y Soler, 2013).

b. A su vez, como el alumnado comparte la meta de resolver un ejercicio interaccionando y llegando a acuerdos con los compañeros y compañeras desarrolla habilidades sociales como el entendimiento y el acuerdo, lo que permite favorecer mejores relaciones de convivencia (Bickmore y Parker, 2014). Las situaciones que obligan a argumentar, contra-argumentar, seleccionar el mejor argumento, ceder, etc. permiten desarrollar un contexto de trabajo colaborativo y armónico (Elboj y Niemelä, 2010; Valls y Kyriakides, 2013; Álvarez y Puigdellívol, 2014).

c. Por otro lado, dado que las tareas se resuelven en un contexto de trabajo activo, donde se producen intercambios ricos en argumentos, los aprendizajes que se generan son más efectivos y duraderos que cuando el alumnado carece de este proceso porque cuando en el futuro deba enfrentarse en solitario a la resolución de tareas semejantes puede rememorar las interacciones mantenidas en el grupo y servirse de estrategias de resolución de ejercicios puestas en juego por los diferentes miembros del equipo (Wells y Arauz, 2006; Mercer y Howe, 2012; Teo, 2013; Gillies, 2014).

d. Dada la forma de trabajo y los principios en que se sustenta esta práctica también es posible identificar en los grupos interactivos un alto potencial en el desarrollo y consolidación de valores relevantes (respeto a diferentes opiniones, tolerancia a la diversidad, solidaridad, compañerismo, violencia cero, etc.) que se fijan en los niños y niñas, al ser vividos en primera persona (Christie, Tolmie, Thurston, Howe y Topping, 2009; Remedios, Clarke y Hawthorne, 2012).

Por todo ello, es posible concluir que en grupos interactivos el alumnado dialoga, toma decisiones por consenso, genera acuerdos en base a argumentos y aprende, lográndose además acelerar/optimizar el aprendizaje. Por todo ello, consideramos que grupos interactivos es una práctica escolar relevante que puede promover el cambio de los centros educativos y la mejora escolar del alumnado dadas sus importantes aportaciones.

Pensamos que la clave del éxito de esta práctica guarda relación con su capacidad para poner en juego formas de enseñanza y aprendizaje apoyadas en teorías y principios valiosos a nivel internacional. Sin embargo, en el futuro serán necesarios más estudios en esta línea, analizando más casos, con carácter longitudinal, para seguir avanzando en el conocimiento de esta actuación educativa.

\section{Referencias}

Álvarez, C. \& Puigdellívol, I. (2014). Cuando la comunidad entra en la escuela: Un estudio de casos sobre los grupos interactivos, valorados por sus protagonistas. Profesorado, 18(3), 239-253.

Aubert, A., Flecha, A., García, C., Flecha, R. \& Racionero, S. (2008). Aprendizaje dialógico en la Sociedad de la Información. Barcelona: Hipatia. 
Bennett-Conroy, W. (2013). Engaging Parents of Eighth Grade Students in Parent-Teacher Bidirectional Communication. School Community Journal, 22(2), 87-110.

Bickmore, K., \& Parker, C. (2014). Constructive conflict talk in classrooms: divergent approaches to addressing divergent perspectives. Theory \& Research in Social Education, 42(3), 291-335.

Brooks, S.M. (2009). A Case Study of School-Community Alliances that Rebuilt a Community. School Community Journal, 19(2), 59-80.

Bosacki, S., Rose-Krasnor, L., \& Coplan, R. J. (2014). Children's talking and listening within the classroom: teachers' insights. Early Child Development and Care, 184(2), 247-265.

Chocarro, E. \& Sáenz, M. (2016). Grupos interactivos: estrategia para la mejora de la convivencia, la participación y el aprendizaje. Revista Complutense de Educación, 27(2), 585-601.

Christie, D., Tolmie, A., Thurston, A., Howe, C., \& Topping, K. (2009). Supporting group work in Scottish primary classrooms: improving the quality of collaborative dialogue. Cambridge Journal of Education, 39(1), 141-156.

Díez, J., Gatt, S., \& Racionero, S. (2011). Placing Immigrant and Minority Family and Community Members at the School's Centre: the role of community participation. European Journal of Education, 46(2), 184-196.

Durand, T.M., (2013). Continuity and Variability in the Parental Involvement and Advocacy Beliefs of Latino Families of Young Children: Finding the Potential for a Collective Voice. School Community Journal, 23(1), 49-79.

Elboj, C., \& Niemela, R. (2010). Sub-communities of mutual learners in the classroom: the case of Interactive groups. Revista de psicodidáctica, 15(2), 177-189.

Elboj, C., Puigdellívol, I., Soler, M. \& Valls, R. (2006). Comunidades de aprendizaje. Transformar la educación. Barcelona: Graó.

Fernández, E. (2015). Las personas voluntarias en los grupos interactivos. Padres y maestros, 362, 3338.

Ferrada, D. \& Flecha, R. (2008). El modelo dialógico de la pedagogía: un aporte desde las experiencias de comunidades de aprendizaje. Estudios pedagógicos, 34(1), 41-61.

Flecha, R. (1997). Compartiendo palabras. El aprendizaje de las personas adultas a través del diálogo. Barcelona: Paidós.

Flecha, R. (2009). Cambio, inclusión y calidad en las comunidades de aprendizaje. Cultura y Educación, 21(2), 157-169.

Flecha, R., \& Soler, M. (2013). Turning difficulties into possibilities: engaging roma families and students in school through dialogic learning. Cambridge Journal of Education, 43(4), 451-465.

Freire, P. (2003). Pedagogía del oprimido. Madrid: Siglo XXI.

García, L., \& Ríos, O. (2014). Participation and family education in school: Successful educational actions. Studies in the Education of Adults, 46(2), 177-191.

Gatt, S., Ojala, M., \& Soler, M. (2011). Promoting social inclusion counting with everyone: Learning Communities and INCLUD-ED. International Studies in Sociology of Education, 21(1), 33-47.

Gillies, R.M. (2014). Developments in classroom-based talk. International Journal of Educational Research, 63, 63-68.

Gómez, M.T., Aguilera, A. \& Prados, M.M. (2016). El voluntariado de la Universidad de Sevilla en los grupos interactivos de las comunidades de aprendizaje. Profesorado: Revista de curriculum y formación del profesorado, 20(2), 278-295.

González, N., \& Moll, L. (2002). Cruzando el Puente: Building Bridges to Funds of Knowledge. EducationalPolicy, 16(4), 623-641.

Habermas, J. (1987). Teoría de la Acción Comunicativa. Madrid: Taurus.

Hammersley, M., \& Atkinson, P. (2007). Ethnography. Principles in practice. London: Routledge. 
Kyle, D.W. (2011). Families' Goals, School Involvement, and Childrens' Academic Achievement: A Follow-up Study Thirteen Years Later. The School Community Journal, 21(2), 9-24.

Lavín, K. (2014). Desarrollo del pensamiento científico por medio de la metodología de grupos interactivos. REXE: Revista de Estudios y Experiencias en Educación, 13(26), 67-80.

Luckacs, K.S., \& Galluzzo, G.R. (2014). Beyond empty vessels and bridges: toward defining teachers as the agents of school change. Teacher Development, 18(1), 100-106.

Ministerio de Educación (2011). Actuaciones de éxito en las escuelas europeas. Madrid: Secretaría general Técnica.

Mello, R. (2009). Diálogo y escuela en Brasil: comunidades de aprendizaje. Cultura y Educación, 21(2), 171-182.

Mercer, N., \& Howe, C. (2012). Explaining the dialogic processes of teaching and learning: The value and potential of sociocultural theory. Learning, Culture and Social Interaction, 1(1), 12-21.

Muntaner, J.J., Pinya, C. \& De la Iglesia, B. (2015). Evaluación de los grupos interactivos desde el paradigma de la educación inclusiva. Revista Electrónica Interuniversitaria de Formación del Profesorado, 18(1), 141-159.

Vieira, L. \& Puigdellívol, I. (2013). ¿Voluntarios dentro del aula? El rol del voluntariado en "Comunidades de Aprendizaje". REXE. Revista de Estudios y Experiencias en Educación, 12(4), 37-55.

Paredes, J. \& De la Herrán, A. (Coords.) (2009). La práctica de la innovación educativa. Madrid: Síntesis.

Racionero, S. (2010). Egalitarian dialogue and instrumental dimension. Two principles of dialogic learning in the classroom. Psychology, Society, \& Education, 2(1), 71-82.

Remedios, L., Clarke, D., \& Hawthorne, L. (2012). Learning to listen and listening to learn: one student's experience of small group collaborative learning. The Australian Educational Researcher, 39(3), 333-348.

Reznitskaya, A., Kuo, L.J., Clark, A.M., Miller, B., Jadallah, M., Anderson, R.C., \& Nguyen-Jahiel, K. (2009). Collaborative reasoning: A dialogic approach to group discussions. Cambridge Journal of Education, 39(1), 29-48.

Stake, R. E. (2005). The art of case study research. London: Sage.

Teo, P. (2013). 'Stretch your answers': Opening the dialogic space in teaching and learning. Learning, Culture and Social Interaction, 2(2), 91-101.

Valls, R., \& Kyriakides, L. (2013). The power of Interactive Groups: how diversity of adults volunteering in classroom groups can promote inclusion and success for children of vulnerable minority ethnic populations. Cambridge Journal of Education, 43(1), 17-33.

Vigotsky, L. (1979). El desarrollo de los procesos psicológicos superiores. Barcelona: Crítica.

Wells, G., \& Arauz, R.M. (2006). Dialogue in the classroom. The journal of the learning sciences, 15(3), $379-428$.

\section{Información sobre los autores}

Autor: Carmen Álvarez-Álvarez

Institución: Facultad de Educación. Universidad de Cantabria.

Email: alvarezmc@unican.es 


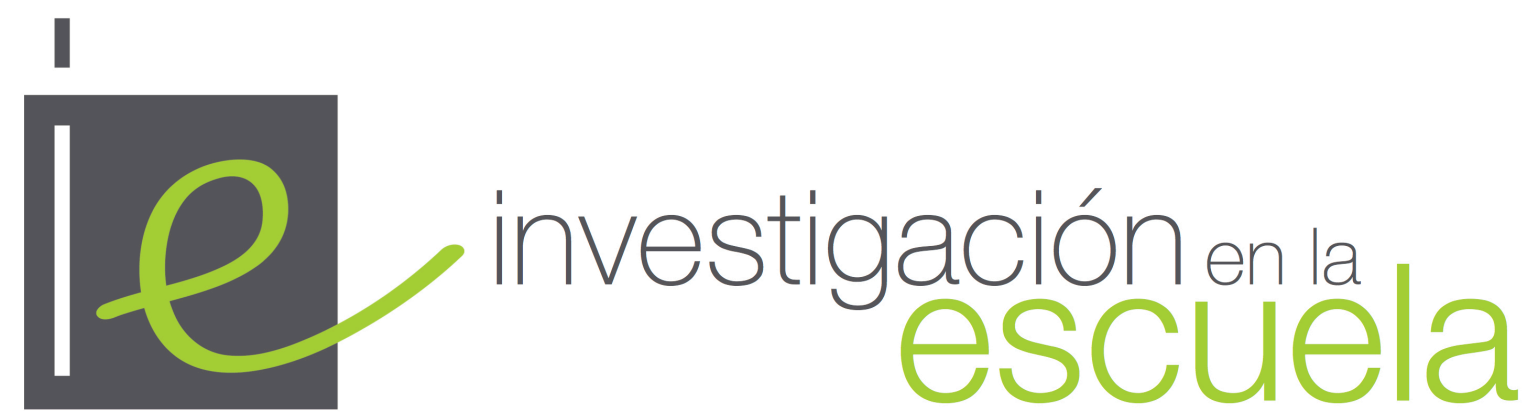

Revista internacional de investigación e innovación educativa

Número 91

06 de julio de 2017

ISSN 2443-9991

\section{(c)}

SORERIIGHISRESERVEDLOS/as lectores/as pueden copiar, mostrar, y distribuir este artículo, siempre y cuando se de crédito y atribución al autor/es y a Investigación en la Escuela, se distribuya con propósitos no-comerciales, no se altere o transforme el trabajo original. Más detalles de la licencia de CreativeCommons se encuentran en http://creativecommons.org/licenses/by-nc-sa/3.0 Cualquier otro uso debe ser aprobado en conjunto por el autor/es, o Investigación en la Escuela.

ํำ investigacion-en-la-escuela

Contribuya con comentarios y sugerencias en la web de la revista. Por errores y sugerencias contactea investigacionescuela@ddcc.uhu.es 


\section{Investigación en la escuela}

Consejo de dirección: Ana Rivero García (Universidad de Sevilla), Nicolás de Alba Fernández(Universidad de Sevilla), Pedro Cañal de León (Universidad de Sevilla), Francisco F. García Pérez (Universidad de Sevilla), Gabriel Travé González, (Universidad de Huelva), Francisco F. Pozuelos Estrada (Universidad de Huelva)

Dirección: Ana Rivero García y Nicolás de Alba Fernández

Secretaría de edición: Elisa Navarro Medina

\section{Consejo editorial}

José Félix Angulo Rasco. Universidad de Cádiz Rosa Má Ávila Ruiz. Universidad de Sevilla Pilar AzcárateGoded. Universidad de Cádiz Juan Bautista Martínez Rodríguez. Universidad de Granada

Nieves Blanco García. Universidad de Málaga Fernando Barragán Medero. Universidad de La Laguna José Carrillo Yáñez. Universidad de Huelva José Contreras Domingo. Universidad de Barcelona. Luis C. Contreras González. Universidad de Huelva Ana $\mathbf{M}^{\mathbf{a}}$ Criado García-Legaz. Universidad de Sevilla Rosario Cubero Pérez. Universidad de Sevilla José $\mathbf{M}^{\mathbf{a}}$ Cuenca López. Universidad de Huelva Jesús Estepa Giménez. Universidad de Huelva Rafael Feito Alonso. Universidad Complutense (Madrid)

Francisco José García Gallardo. Universidad de Huelva

Soledad García Gómez. Universidad de Sevilla J. Eduardo García Díaz. Universidad de Sevilla
Fernando Hernández Hernández. Universidad de Barcelona

Salvador Llinares Ciscar. Universidad de Alicante Alfonso Luque Lozano. Universidad de Sevilla Rosa Martín del Pozo. Universidad Complutense (Madrid)

José Martín Toscano. IES Fernando Herrera (Sevilla) Jaume Martínez Bonafé. Universidad de Valencia F. Javier Merchán Iglesias. Universidad de Sevilla Emilia Moreno Sánchez. Universidad de Huelva.Rosario Ortega Ruiz. Universidad de Córdoba

Antonio de Pro Bueno. Universidad de Murcia Fco. de Paula Rodríguez Miranda. Universidad de Huelva

Pedro Sáenz-López Buñuel. Universidad de Huelva Antoni Santisteban Fernández. Universidad Autónoma (Barcelona) Emilio Solís Ramírez. Catedrático de IES. $\mathbf{M}^{\mathbf{a}}$ Victoria Sánchez García. Universidad de Sevilla. Magdalena Suárez Ortega. Universidad de Sevilla

\section{Consejo asesor}

Manuel Área Moreira. Universidad de La Laguna

Jaume Carbonell. Director Cuadernos de Pedagogía. Barcelona

César Coll. Universidad de Barcelona

Christopher Day. Universidad de Nothingham. U.K.

Juan Delval. Universidad Nacional de Educación a Distancia

John Elliott. Universidad de East Anglia. Norwich. U.K.

José Gimeno Sacristán. Universidad de Valencia

André Giordan. Universidad de Paris VII y Ginebra

Francisco Imbernón. Universidad de Barcelona

Ángel Pérez Gómez. Universidad de Málaga

Rafael Porlán Ariza. Universidad de Sevilla

Francesco Tonucci. Instituto de Pedagogía del C.N.R. Roma

Jurjo Torres Santomé. Universidad de A Coruña 
